\title{
Unveiling the early events of Pseudomonas aeruginosa adaptation in cystic fibrosis airway environment using a long-term in vitro maintenance
}

\author{
Ana Margarida Sousa, Rosana Monteiro, Maria Olívia Pereira* \\ CEB - Centre of Biological Engineering, LIBRO - Laboratório de Investigação em Biofilmes Rosário Oliveira, University of Minho, Campus de Gualtar, $4710-057$ Braga, \\ Portugal
}

\section{A R T I C L E I N F O}

\section{Keywords:}

Cystic fibrosis

Pseudomonas aeruginosa

Long-term in vitro model

Early infection

Phenotypic diversification

\begin{abstract}
A B S T R A C T
Pseudomonas aeruginosa chronic infections are the major cause of high morbidity and mortality in cystic fibrosis (CF) patients due to the use of sophisticated mechanisms of adaptation, including clonal diversification into specialized CF-adapted phenotypes. In contrast to chronic infections, very little is known about what occurs after $\mathrm{CF}$ lungs colonization and at early infection stages.

This study aims to investigate the early events of $P$. aeruginosa adaptation to CF environment, in particular, to inspect the occurrence of clonal diversification at early stages of infection development and its impact on antibiotherapy effectiveness.

To mimic CF early infections, three $P$. aeruginosa strains were long-term grown in artificial sputum (ASM) over 10 days and phenotypic diversity verified through colony morphology characterization. Biofilm sub- and inhibitory concentrations of ciprofloxacin were applied to non- and diversified populations to evaluate antibiotic effectiveness on $P$. aeruginosa eradication.

Our results demonstrated that clonal diversification might occur after ASM colonization and growth. However, this phenotypic diversification did not compromise ciprofloxacin efficacy in $P$. aeruginosa eradication since a biofilm minimal inhibitory dosage would be applied. The expected absence of mutators in $P$. aeruginosa populations led us to speculate that clonal diversification in the absence of ciprofloxacin treatments could be driven by niche specialization. Yet, biofilm sub-inhibitory concentrations of ciprofloxacin seemed to overlap niche specialization as "fitter" variants emerged, such as mucoid, small colony and pinpoint variants, known to be highly resistant to antibiotics. The pathogenic potential of all emergent colony morphotypes-associated bacteria, distinct from the wild-morphotypes, revealed that $P$. aeruginosa evolved to a non-swimming phenotype. Impaired swimming motility seemed to be one of the first evolutionary steps of $P$. aeruginosa in CF lungs that could pave the way for further adaptation steps including biofilm formation and progress to chronic infection. Based on our findings, impaired swimming motility seemed to be a candidate to disease marker of $P$. aeruginosa infection development. Despite our in vitro CF model represents a step forward towards in vivo scenario simulation and provided valuable insights about the early events, more and distinct $P$. aeruginosa strains should be studied to strengthen our results.
\end{abstract}

\section{Introduction}

Cystic fibrosis $(\mathrm{CF})$ is an autosomal recessive disease caused by a defect in the cystic fibrosis conductance regulator gene resulting in several complications in the human body (Kreda et al., 2012). So far, the pathological changes in the lungs are the best studied due to the high mortality rates linked to the poor lung function and the recurrent development of microbial infections (Flume et al., 2009; Ciofu et al., 2014). CF lungs are often colonized or infected with a complex microbial flora, mainly composed by bacteria, provoking acute and chronic infections (Bittar et al., 2008; Coburn et al., 2015). Pseudomonas aeruginosa is the most common pathogen isolated from $\mathrm{CF}$ sputum and its presence is highly associated with increased exacerbations, decline of the lung function and patients death (Bittar et al., 2008; Coutinho et al., 2008; Folkesson et al., 2012).

In attempt to eradicate $P$. aeruginosa infections, numerous antibiotic strategies have been used varying in the route of drug administration (systemic, oral, inhaled antibiotics or route combination), classes of antibiotics and treatment duration (Schelstraete et al., 2013; Langan et al., 2015). Despite these advances and the long and aggressive

\footnotetext{
* Corresponding author at: Centre of Biological Engineering, University of Minho, 4710-057, Braga, Portugal.

E-mail address: mopereira@deb.uminho.pt (M.O. Pereira).
} 
antibiotic treatments, $P$. aeruginosa is still able to persist causing chronic infections almost impossible to eradicate (Mayer-Hamblett et al., 2012). This long-term persistence of $P$. aeruginosa in CF lungs has been associated with its clonal diversification, or expansion, into specialized phenotypes. During infection progression in CF lungs, $P$. aeruginosa switches from an acute environmental virulent pathogen, characteristic of early infection stages, to a CF-adapted pathogen, typical of chronic infection stages (Sousa and Pereira, 2014). The microbiological analysis of chronic isolates revealed changes in several $P$. aeruginosa phenotypic traits such as colony morphology with distinct consistency, size, texture and colour, inactivation of quorum sensing, hypermutability, loss of Oantigen components of the lipopolysaccharide, loss of motility, resistance to antibiotics, changes in nutritional requirements and other virulence-associated traits (Bragonzi et al., 2009; Rau et al., 2010; Workentine et al., 2013; Jiricny et al., 2014). In fact, some of these factors have been considered the hallmark of CF disease and can even be indicative of the chronic infection stage, such as the alteration of colony morphology to the mucoid phenotype and to small colony variants (SCV).

Mucoid colony morphology results from alginate overproduction, absence of flagellin and pilin and expression of other virulence factors (Hogardt and Heesemann, 2010). Within the mucoid form, P. aeruginosa is more difficult to eradicate because alginate promotes its encapsulation and biofilm formation, protecting bacteria from the action of reactive oxygen species, antibiotics and host immune defences, and assisting its persistence in CF lungs (Qiu et al., 2007; Hassett et al., 2009; Høiby et al., 2010).

$P$. aeruginosa SCV, the small-sized colonies typically with $1-3 \mathrm{~mm}$ of diameter after $24-48 \mathrm{~h}$ of growth, are also frequently isolated from chronic CF lung infections, mainly after long antibiotic courses (Haussler et al., 1999; Kahl, 2014). These colony variants are normally hyperpiliated, hyperadherent, excellent biofilm formers and exhibit autoaggregative behavior and increased twitching motility (Deziel et al., 2001; Haussler et al., 2003; Haussler, 2004; Kirisits et al., 2005). Moreover, SCV display augmented resistance to several classes of antibiotics, notably to aminoglycosides, contributing to $P$. aeruginosa persistence in CF airways and decline of lung function (Haussler et al., 1999; Kahl, 2014). Other colony morphologies have also been isolated from CF airways but their roles in $P$. aeruginosa persistence and in infection progression in CF lungs remain unclear so far (Rakhimova et al., 2008; Workentine et al., 2013; Clark et al., 2015).

When $P$. aeruginosa is identified, the gold standard of CF disease management is its eradication from CF lungs as soon as detected (Ratjen et al., 2010; Emiralioglu et al., 2016; Ratjen et al., 2018). Early eradication of $P$. aeruginosa is of upmost importance as it avoids or, at least, retards the development of chronic infections preserving lung function. However, some antibiotics fail in early eradication of $P$. aeruginosa giving rise to serious complications, as the emergence of resistant subpopulations, which can pave the way for the establishment of a chronic infection (Ratjen, 2006; Mayer-Hamblett et al., 2014a,b). Therefore, early antibiotic treatment must be adequately designed in order to obtain successful $P$. aeruginosa eradication. However, very little is known about the early adaptations that $P$. aeruginosa underwent after CF lungs colonization and which impact they have on the effectiveness of the first antibiotic treatments. Without understanding the early events of $P$. aeruginosa adaptation to CF lungs, improving antimicrobial stewardships could be a goal hard to achieve. In the light of this knowledge gap, this study put special emphasis on the monitoring of initial $P$. aeruginosa colonization and early growth within CF artificial sputum using a novel in vitro $\mathrm{CF}$ model. The main goal of this study was to understand the early adaptation mechanisms that $P$. aeruginosa uses to adapt and grow in CF environment and also to evade the action of antimicrobial agents, events up to now unclear. In particular, this study intends to inspect the occurrence of clonal diversification, after simulated $C F$ lungs colonization by $P$. aeruginosa, to gain insights into the driving forces that can trigger diversification and, also, to determine the impact of clonal diversification in antibiotic effectiveness.

\section{Materials \& methods}

\subsection{Bacterial strains and culture conditions}

P. aeruginosa PAO1, ATCC ${ }^{\circledR} 39324$ and a clinical isolated PAI were used throughout this study. The rationale behind the selection of the strains was based on the source and routes of $P$. aeruginosa transmission to CF patients. Most of the CF patients are colonized by environmental strains (Burns et al., 2001; Speert et al., 2002), but also via contact with other non- and CF patients (UK Cystic Fibrosis Trust, 2004). PAO1 is a non-mucoid laboratory strain that never contacted with $\mathrm{CF}$ environment (Stover et al., 2000). PAI is a respiratory clinical isolate non-CF related kindly provided by the Hospital of Braga (Braga, Portugal) (Pires et al., 2011). P. aeruginosa ATCC ${ }^{\circledR} 39324$, from now on referred as ATCC, is a CF strain able to produce an exo-polysaccharide conferring mucoidy appearance (data obtained from ATCC $^{\circledR}$ product sheet). Bacteria were routinely cultured on Trypic Soy Broth (TSB, Liofilchem) or Trypic Soy Agar (TSA, Liofilchem) at $37^{\circ} \mathrm{C}$. All strains were preserved in criovials (Nalgene) at $-80 \pm 2{ }^{\circ} \mathrm{C}$ to minimise putative adaptation to the laboratory environment. Prior to each experiment, bacterial cells were grown on TSA plates overnight at $37^{\circ} \mathrm{C}$.

\subsection{P. aeruginosa biofilm-cells susceptibility to ciprofloxacin}

Biofilms were developed as previously described (Stepanovic et al., 2000). Briefly, overnight cultures of each strain, grown in TSB at $37^{\circ} \mathrm{C}$ and $120 \mathrm{rpm}$ in air conditions, were washed in sterile water and diluted in TSB to obtain $1 \times 10^{7} \mathrm{CFU} / \mathrm{mL}$ as final concentration. Triplicate wells of a 96-well polystyrene microtiter plate (Orange Scientific) were inoculated with $200 \mu \mathrm{L}$ of the bacterial suspensions to form biofilms for $24 \mathrm{~h}$ at $37^{\circ} \mathrm{C}$ on a horizontal shaker $(120 \mathrm{rpm})$. After that, the liquid content was withdrawn and biofilms washed twice with sterile water to remove weakly adhered cells. Plates were then filled with sterile water and placed on an ultrasound bath (Sonic model SC-52, UK) for $6 \mathrm{~min}$ (time previously optimized by Magalhães et al. (2016)) to detach biofilm-cells. Then, biofilm-cells suspensions were gently vortexed to homogenize and used to assess the susceptibility of the biofilm-derived cells towards ciprofloxacin (Fluka), testing a range of concentrations from 0,125 to $16 \mathrm{mg} / \mathrm{L}$.

The ciprofloxacin susceptibility profiles of the biofilm-cells were established determining the minimum inhibitory concentration (MIC) by microdilution broth assay following the recommendations of the CLSI (Clinical and Laboratory Standards Institute, 2007). Briefly, sterile 96-well round-bottom microtiter plates (polystyrene, Orange, USA) were filled in with $100 \mu \mathrm{L}$ of Muller-Hinton Broth (Liofilchem) with increasing concentrations of ciprofloxacin and further $100 \mu \mathrm{L}$ of each biofilm-cell suspension were added at a final concentration of $5 \times 10^{5}$ $\mathrm{CFU} / \mathrm{mL}$. The plates were afterwards incubated at $37^{\circ} \mathrm{C}$ for $18-21 \mathrm{~h}$ in an orbital shaker at $120 \mathrm{rpm}$. In this assay, two controls were used, one without bacteria, as a negative control and one without ciprofloxacin, as a positive control. The MIC was obtained by measuring the absorbance at $640 \mathrm{~nm}\left(\mathrm{~A}_{640 \mathrm{~nm}}\right)$, where clear wells $\left(\mathrm{A}_{640 \mathrm{~nm}}=\right.$ negative control) were evidence of bacterial growth inhibition.

\subsection{In vitro growth of $P$. aeruginosa in an artificial CF lung environment}

\subsubsection{Artificial sputum medium preparation}

Artificial sputum medium (ASM) was used to mimic the sputum of CF patients and it was prepared as described by Sriramulu et al. (2005). Briefly, $5 \mathrm{~g} / \mathrm{L}$ of mucin from pig stomach (Sigma-Aldrich), $4 \mathrm{~g} / \mathrm{L}$ of DNA from salmon sperm (Sigma-Aldrich), $5.9 \mathrm{mg} / \mathrm{L}$ of diethylene triamine pentaacetic acid (DTPA, Sigma-Aldrich), $5 \mathrm{~g} / \mathrm{L}$ of $\mathrm{NaCl}, 2.2 \mathrm{~g} / \mathrm{L}$ of $\mathrm{KCl}$ and $5 \mathrm{~g} / \mathrm{L}$ of casoamino acids (AMESRO) were resuspended in water and the $\mathrm{pH}$ adjusted to 7 with Tris base. This ASM was then sterilized in 
an autoclave at $110^{\circ} \mathrm{C}$ for $15 \mathrm{~min}$ and, after cooled, $5 \mathrm{~mL}$ of egg yolk emulsion (Fluka) were added.

\subsection{Long-term in vitro maintenance model of $P$. aeruginosa in ASM}

Overnight $P$. aeruginosa inocula of each bacterial strain were washed twice in sterile water by centrifugation $(9000 \mathrm{~g}, 5 \mathrm{~min}$ ) and further serial diluted in sterile water to a final concentration of $4 \times 10^{9} \mathrm{CFU} / \mathrm{mL}$. Two $\mathrm{mL}$ of ASM were transferred to each well of a 24 well-plate (polystyrene, Orange, USA) and inoculated on the top with $5 \mu \mathrm{L}$ of the bacterial cell suspensions, obtaining a final cellular concentration in each well of $1 \times 10^{7} \mathrm{CFU} / \mathrm{mL}$. ASM cultures were incubated at $37^{\circ} \mathrm{C}$ aerobically for 10 days, to mimic the early stage of CF lung disease, and in static culture conditions to resemble the reduced or absent cilia movements in CF lungs (Worlitzsch et al., 2002; Hassett et al., 2009). After 1, 3, 5, 7 and 10 days of bacterial growth in ASM, the content of the wells was collected aseptically and vigorously shaken to detach cells from the small aggregates or cells adhered to mucin. These experiments were performed at least 5 times. The resulting cell suspensions of each sampling time were analysed in terms of cell viability and phenotypic diversification.

\subsection{Application of ciprofloxacin treatments to ASM-grown populations}

To investigate the impact of early phenotypic diversification in the effectiveness of antibiotic treatment, two strategies of ciprofloxacin application were followed. First, ciprofloxacin was applied to non-diversified $P$. aeruginosa populations, being thus added to the top of ASM cultures $30 \mathrm{~min}$ after bacterial inoculation and every $24 \mathrm{~h}$ over 10 days. The second therapeutic strategy was applied against phenotypic diversified populations adding ciprofloxacin to ASM cultures after 5 days of bacterial growth (when diversification was observed) and every $24 \mathrm{~h}$ over the last 5 days. For each strategy, two different concentrations were used, a sub- and a biofilm inhibitory concentration, according to the MIC outcomes determined for biofilm-cells of each of the three strains. For both strategies, ASM-grown populations were studied as above described. All experiments were performed at least 5 times.

\subsection{Cell viability determination}

The viability of the bacterial cells was determined through colonyforming unit (CFU). For CFU counts, both non- and treated cell suspensions were serial diluted, plated on TSA and incubated overnight at $37^{\circ} \mathrm{C}$. After that, the number of colonies was counted and verified after more $24 \mathrm{~h}$ of growth to ensure that slow growing bacteria were reckoned. These CFU counts were used to plot the growth kinetics of the $P$. aeruginosa strains grown in ASM and the time killing curves for ciprofloxacin.

\subsection{Phenotypic diversification during bacterial growth in ASM}

The occurrence of $P$. aeruginosa clonal diversification was assessed through colony morphology inspection over time. $P$. aeruginosa populations grown in ASM were allowed to grow on TSA plates during $48 \mathrm{~h}$ at $37^{\circ} \mathrm{C}$ in air conditions as previously optimized by Sousa et al. (2013), ensuring thus that the conditions used to observe phenotypic diversity were fully controlled and identical in all over the experiments. Colony morphologies were observed by directly placing the Petri plates on a magnifying glass (Olympus SZ-CTV) and photographed in a CCD camera (AVC, D5CE; Sony, Tokio, Japan). The morphological features of the colonies were annotated using the morphological criteria previously stipulated (Sousa et al., 2015). In brief, P. aeruginosa colonies were evaluated according to their form, margin, type of surface, texture, size, sheath, elevation, opacity, consistency and colour. Wildcolony morphotype (WMT) of each $P$. aeruginosa strain was established studying inoculum cultures plated in the same abovementioned conditions. The distinct colony morphotypes (MT) identified were referenced with an identity number, for instance MT01. The identity numbers had not any correlation with the order of emergence or relationships with other colony morphotypes within populations, being used only to refer colonies with common features and distinguish them from each other. The estimated relative abundance of each colony morphotype was calculated based on the proportion of a morphotype in our photographically records of the population.

The different morphotype-forming bacteria were characterized according to the virulence determinants more relevant in the CF context, namely biofilm formation, motility, auxotrophy, pigments and hemolysin production and their antibiotic susceptibility.

\subsection{Pathogenic potential of the different colony morphotype-forming bacteria}

\subsubsection{Biofilm formation}

The ability of colony morphotype-associated bacteria to form biofilms was assessed by the crystal violet (CV) method (Peeters et al., 2007). Colony morphotypes were suspended and diluted in TSB to obtain a final concentration of $1 \times 10^{7} \mathrm{CFU} / \mathrm{mL}$. This cell suspension was transferred to 96 -well microtitre plates $(200 \mu \mathrm{L}$ per well) being then incubated at $37^{\circ} \mathrm{C}$ for $24 \mathrm{~h}$ under agitation $(120 \mathrm{rpm}$ ) in air conditions. After $24 \mathrm{~h}$ of biofilm formation, the liquid content of the plates was discarded and the adhered biomass washed twice with sterile water in order to remove weakly attached cells. Afterwards, $200 \mu \mathrm{L}$ per well of methanol were added and plates were allowed to stand for $15 \mathrm{~min}$ in order to fix the biofilm-mass. Methanol was discarded and plates were left to dry at room temperature. Biofilms were stained with $200 \mu \mathrm{L}$ of pure CV for approximately $5 \mathrm{~min}$ and then rinsed thoroughly and repeatedly with tap water. Finally, the amount of biofilm was quantified by solubilization of the CV in $200 \mu \mathrm{L}$ of $33 \%$ (v/v) acetic acid. The absorbance was measured at $570 \mathrm{~nm}$ using a microtiter plate reader (Bio-Rek Synergy HT, Izasa).

\subsubsection{Motility}

Swimming, swarming and twitching were assessed using procedures previously described (Manos et al., 2013). Briefly, swimming, swarming and twitching motilities were analysed using TSA plates with $0.3 \%$, $0.5 \%$ and $1.5 \%(\mathrm{w} / \mathrm{v})$ of agar, respectively, inoculated with $1 \times 10^{8}$ $\mathrm{CFU} / \mathrm{mL}$ of each colony morphotype-associated bacteria and incubated for $24 \mathrm{~h}$ at $37^{\circ} \mathrm{C}$ in air conditions. Swimming motility was assessed by measuring the circular turbid zone around the inoculation point, swarming by measuring the irregular branching that appeared at the periphery of the colonies and twitching by measuring the hazy zone of growth at the interface between the agar and the polystyrene surface.

\subsubsection{Auxotrophy testing}

This metabolic trait was investigated by determining the ability of colony morphotypes-associated bacteria to grow on glucose M9 medium (AMESRO) with and without $0.2 \%(\mathrm{w} / \mathrm{v})$ of casoamino acids (Workentine et al., 2013). Colony morphotypes were transferred to M9 medium plates with and without casoamino acids and incubated at $37^{\circ} \mathrm{C}$ and let grow for 24 and $48 \mathrm{~h}$. Absence of growth on M9 media without casoamino acids was indicative of auxotrophy.

\subsubsection{Pigments production}

Pigments production was inferred visually observing the colour of colonies and the surrounding agar medium. Colonies exhibiting bluegreen, green, red and brown colours on TSA plates were considered pyocyanin, pyoverdine, pyorubrin, pyomelanin producers (MayerHamblett et al., 2014a, b).

\subsubsection{Hemolytic activity}

The hemolytic activity of colony morphotypes-associated bacteria was assessed using Columbia Agar (Liofilchem) with $40 \%$ (v/v) of sheep 
blood (Probiologica) plates (CAB). Colony morphology-associated bacteria were transferred to $\mathrm{CAB}$ plates and incubated $24 \mathrm{~h}$ at $37^{\circ} \mathrm{C}$ in air conditions. Hemolysin production was considered negative when no clearing zones were observed around the colonies.

All the tests were performed at least 5 times and each test used 3 technical replicates to ensure precision of each method.

\subsubsection{Antimicrobial susceptibility testing}

The antimicrobial susceptibility profile of each morphotype was determined using the disk diffusion method for ten antibiotics commonly used to treat $P$. aeruginosa infections in CF context: amikacin (AK) $30 \mu \mathrm{g}$, aztreonam (ATM) $30 \mu \mathrm{g}$, ciprofloxacin (CIP) $5 \mu \mathrm{g}$, tobramycin (TOB) $10 \mu \mathrm{g}$, gentamicin (CN) $10 \mu \mathrm{g}$, ceftazidime (CAZ) $30 \mu \mathrm{g}$, cefepime (FEP) $30 \mu \mathrm{g}$, imipenem (IMI) $10 \mu \mathrm{g}$, piperacillin (PRL) $100 \mu \mathrm{g}$ and ticarcillin/clavulanic acid (TTC) $85(75+10) \mu \mathrm{g}$, all obtained from Liofilchem. Disk diffusion susceptibility testing was performed in accordance with the recommendations of NCCLS document M100-522 (Clinical and Laboratory Standards Institute, 2012). The cartridges of antibiotic disks were stored at $-20^{\circ} \mathrm{C}$ and allowed to come to room temperature prior to use. The inhibition zones were measured (mm) after $18-21 \mathrm{~h}$ at $37^{\circ} \mathrm{C}$ in air conditions. Colony morphotypes-associated bacteria were assigned as resistant (R), intermediate resistant (I) or sensitive (S) as defined by NCCLS guidelines in document M100-S28 (Clinical and Laboratory Standards Institute, 2018).

\subsection{Statistical analysis}

Continuous variables including $\mathrm{CFU} / \mathrm{mL}$ and quantification of biofilm biomass data (absorbance values) were the only variables analysed statistically. CFU data retrieved from growth kinetics and time killing curves were analysed by Mann-Whitney test, a non-parametric procedure to assess a null hypothesis that two populations are the same. In vitro ability to form biofilms data (absorbance values) were analysed using Kruskal Wallis (assumption of normality was not verified). All statistical analyses were performed in SPSS version 20.0 (Chicago, Illinois, USA) considering as level of statistical significance $p<0.05$.

\section{Results}

The main goal of this study was to inspect the possible early adaptations that $P$. aeruginosa underwent after CF lungs colonization using a long-term in vitro maintenance model mimicking $C F$ lung environment. Our model was designed to allow the growth of $P$. aeruginosa in ASM along 10 days in order to provide sufficient time to bacteria initiate their adaptation to $\mathrm{CF}$ environment. Through this in vitro long-term growth in ASM, our study aimed to investigate: i) the occurrence of $P$. aeruginosa diversification after simulated in vitro CF lungs colonization and growth; ii) the driving forces underlying phenotypic diversification; iii) the impact of phenotypic diversification on the effectiveness of the first or early anti-pseudomonal treatments; and iv) the $P$. aeruginosa response to ciprofloxacin long-term exposure. To gain more valuable insights about the early adaptations that $P$. aeruginosa can undergo, three different strains were selected to simulate the main sources and routes of $P$. aeruginosa transmission to CF patients. The majority of $\mathrm{CF}$ patients are colonized by environmental strains (Burns et al., 2001; Speert et al., 2002), which means these $P$. aeruginosa strains never contacted with CF environment or human host. This scenario was simulated using PAO1, a laboratory strain (Stover et al., 2000). During $\mathrm{CF}$ patients visits to hospital or even during their hospitalizations, $\mathrm{CF}$ patients can contact with other non- and $\mathrm{CF}$ individuals that can transmit $P$. aeruginosa (UK Cystic Fibrosis Trust, 2004). To simulate these two scenarios, two $P$. aeruginosa clinical isolates non- and CF related, PAI and ATCC, respectively, were chosen. The inclusion of the ATCC strain also allowed to investigate whether a first CF patient colonization by a mucoid $P$. aeruginosa represents an increased risk to develop a faster chronic infection, issue that stills controversial in

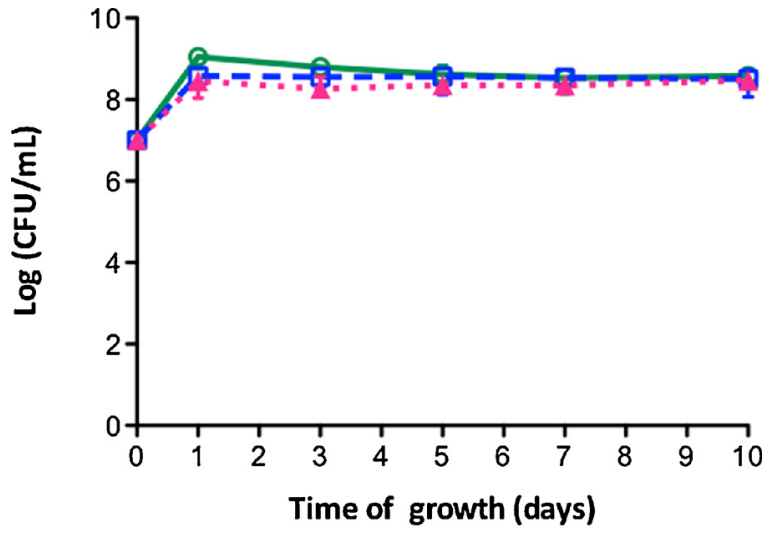

$\because$ PA01 -A-ATCC $\quad-$ PAI

Fig. 1. Growth kinetics of ASM-grown P. aeruginosa strains over 10 days.

clinical community.

3.1. Growth kinetics of ASM-grown populations and time killing curves of ciprofloxacin treatments

The growth kinetics of the three ASM-grown $P$. aeruginosa populations demonstrated identical growth profiles (Fig. 1). After one day of exponential growth, the three ASM-grown populations achieved the stationary phase with no difference in the number of viable cells among all the populations. Regarding phenotypic diversity, results demonstrated the occurrence of early diversification, being the diversification profiles strongly strain-dependent (Fig. 2A). After 3 days of growth in ASM, new colony morphotypes, different from the ones observed in the inoculum (day 0), emerged in P. aeruginosa PAI and ATCC populations (Fig. 3 and S1). In contrast, ASM-grown PAO1 population did not exhibit colony diversity.

$P$. aeruginosa PAI population exhibited the highest diversity since it generated 4 new distinct morphotypes, all catalogued as SCV (MT04, MT05, MT07 and MT10) (Table 1), but the new morphotypes had only reached a maximum of $15 \%$ of estimated prevalence (Figure S1). In turn, ATCC strain generated only one new morphotype, a dry variant (MT12) more prevalent than WMT. Given these evidences of early phenotypic diversification, the mutation frequencies were determined to inspect whether the existence of mutators could be the mechanism underlying diversity. The expected absence of mutators in $P$. aeruginosa populations at these early stages (data not shown) and the coexistence of colony variants within the 10-day period of bacterial growth in ASM pointed out that diversification was not triggered by increased mutation frequencies. Therefore, the results obtained until now led to speculate that $P$. aeruginosa could phenotypically diversify after CF lungs colonization and at early growth stages. Based on this evidence, it was considered of vital clinical significance to know whether this diversification could affect antibiotic effectiveness in $P$. aeruginosa early eradication from CF lungs. Accordingly, different ciprofloxacin treatment approaches were tested against non- and diversified $P$. aeruginosa populations grown in ASM varying antibiotic concentration. The inoculum populations of the three strains used to colonize the ASM (day 0) did not exhibit population diversity (Figs. 2 and 3). Therefore, one of the ciprofloxacin treatment approaches started at day 0 to ensure that antibiotic would act against non-diversified populations. The results obtained from population diversity displayed in Fig. 2A were also crucial to set down the timing of ciprofloxacin application against diversified populations. As ASM-grown PAI and ATCC populations exhibited colony morphology diversity after 3 days of growth, ciprofloxacin treatments against diversified populations were initiated at day 5 of bacterial growth to ensure well-established phenotypic diversity. 


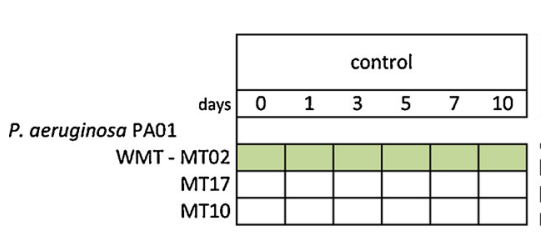

P. aeruginosa ATCC
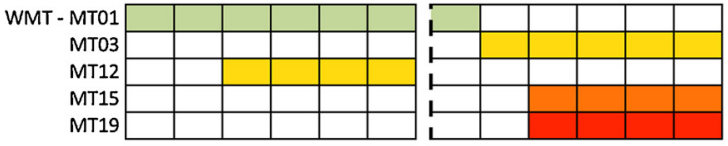

P. aeruginosa PAI
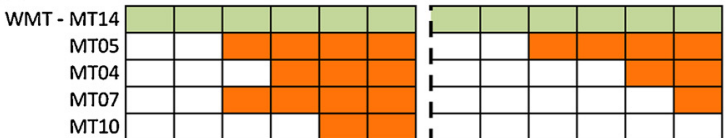

wild-colony morphotype (WMT)

dry colony morphotype

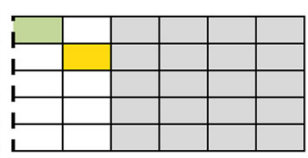

\begin{tabular}{|c|c|c|c|c|c|c|c|c|c|c|c|}
\hline \multicolumn{6}{|c|}{$1 \mathrm{mg} / \mathrm{L}$} & \multicolumn{6}{|c|}{$4 \mathrm{mg} / \mathrm{L}$} \\
\hline 0 & 1 & 3 & 5 & 7 & 10 & 0 & 1 & 3 & 5 & 7 & 10 \\
\hline
\end{tabular}
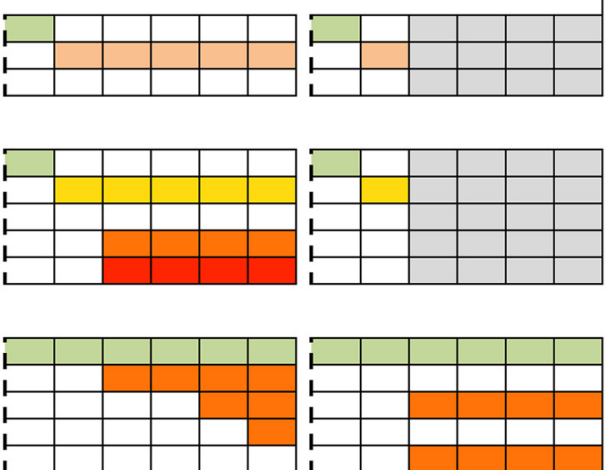

moist colony morphotype SCV
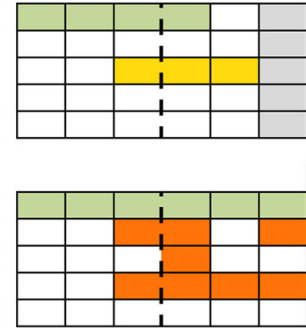

Ciprofloxacin treatment against diversifed population

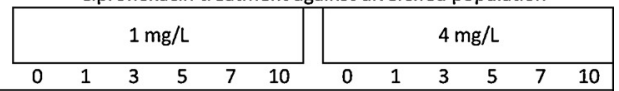
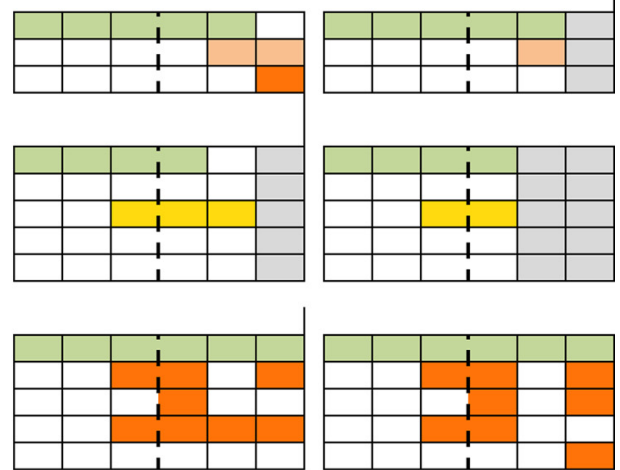

pinpoint variant

bacterial eradication

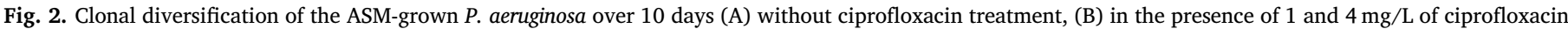

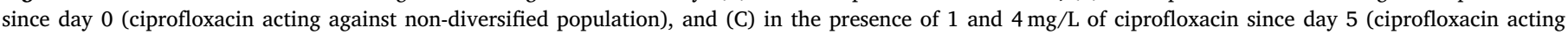
against diversified population). Dash lines indicate when ciprofloxacin treatments were applied.

Despite ASM-grown PAO1 population did not diversify its colony morphology, this strain was included in these experiments to serve as negative control of this mode of ciprofloxacin application. Beyond population diversity, distinct ciprofloxacin concentrations (sub- and biofilm inhibitory) were used to evaluate the dosage-effect on antibiotic effectiveness against non- and diversified $P$. aeruginosa populations. According to Sriramulu et al. (2005), P. aeruginosa growth in ASM promotes the formation of tight microcolonies attached to sputum constituents or even could promote the formation of biofilms. Therefore, the antibiotic susceptibility profiles of ASM-grown P. aeruginosa populations were expected to be similar to the biofilm-cells. By this reason, the ciprofloxacin concentrations were stipulated according to the MIC of $P$. aeruginosa biofilm-derived cells. All $P$. aeruginosa biofilmcells exhibited identical MIC of $4 \mathrm{mg} / \mathrm{L}$ of ciprofloxacin and, thus, $1 \mathrm{mg} /$ $\mathrm{L}$ (sub-inhibitory concentration equivalent of $1 / 4 \mathrm{MIC}$ ) and $4 \mathrm{mg} / \mathrm{L}$ (inhibitory concentration) of ciprofloxacin were chosen to test antibiotic efficacy against ASM-grown $P$. aeruginosa populations. Moreover, the bacterial response to these distinct long-term antibiotic exposures was also investigated.

The thorough analysis of the time killing curves (Fig. 4) highlighted that the use of sub-inhibitory concentrations of $1 \mathrm{mg} / \mathrm{L}$ of ciprofloxacin against non-diversified populations was clearly inefficient in the eradication of $P$. aeruginosa (Fig. 4A). This ciprofloxacin treatment approach seemed to have no significant effect over PAO1 population; promote bacterial load reduction for the ATCC population, where a 3 log reduction was attained; and have no effect against PAI population. Ciprofloxacin efficacy was improved using the inhibitory concentration of $4 \mathrm{mg} / \mathrm{L}$ (Fig. 4B) as ASM-grown PAO1 and ATCC populations were eradicated after three days of treatment, even though no interference in PAI growth was observed. Regarding antibiotic application over the
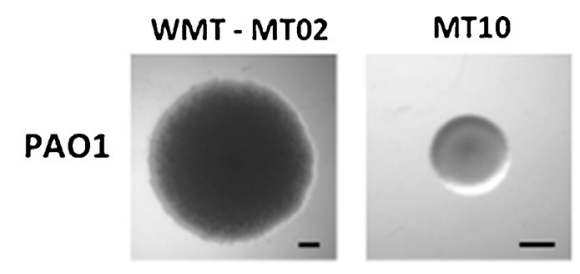

MT17

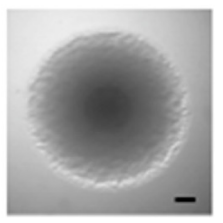

WMT - MT01

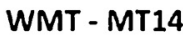

PAI

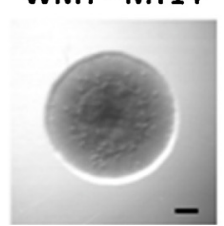

MT03

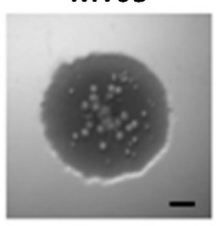

MT04

MT12

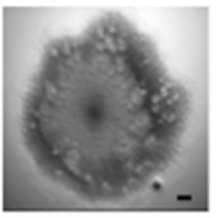

MT05
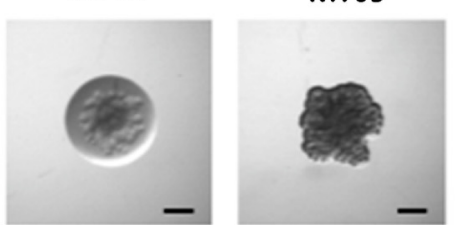

MT15

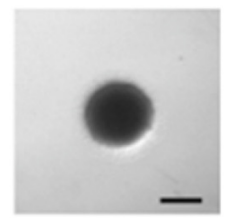

MT07

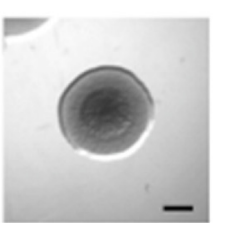

MT19

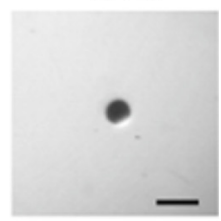

MT10

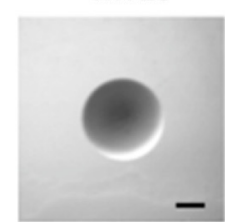

Fig. 3. Colony morphotypes isolated from ASM-grown P. aeruginosa populations over 10 days with and without ciprofloxacin treatment. All colonies were grown on TSA for $48 \mathrm{~h}$. Black bar $=1 \mathrm{~mm}$. 
Table 1

Detailed morphological description of the colonies identified in ASM-grown $P$. aeruginosa populations with and without ciprofloxacin treatment.

\begin{tabular}{|c|c|c|c|c|c|c|c|c|c|c|}
\hline & Form & Margin & Sheath & Type of Surface & Texture & Consistency & Elevation & Opacity & Size & Colour \\
\hline \multicolumn{11}{|l|}{ PA01 } \\
\hline WMT- MT02 & circular & undulate & present & homogeneous & rough & dry & flat & opaque & large & yellow \\
\hline MT17 & circular & undulate & present & homogeneous & rough & moist & flat & opaque & large & yellow \\
\hline \multicolumn{11}{|l|}{ ATCC } \\
\hline WMT - MT01 & circular & undulate & present & homogeneous & smooth & mucoid & flat & opaque & large & beige \\
\hline MT03 & circular & undulate & present & homogeneous & rough & dry & flat & opaque & large & beige \\
\hline MT12 & circular & undulate & present & homogeneous & rough & dry & flat & transparent & large & beige \\
\hline MT15 & circular & undulate & present & homogeneous & rough & dry & flat & opaque & pinpoint & beige \\
\hline MT19 & circular & undulate & present & homogeneous & rough & dry & flat & opaque & small & beige \\
\hline \multicolumn{11}{|l|}{ PAI } \\
\hline WMT - MT14 & circular & entire & absent & homogeneous & rough & dry & flat & iridescent & large & Blue-green \\
\hline MT04 & circular & undulate & absent & heterogeneous & smooth/rough & dry & flat & iridescent & small & Blue-green \\
\hline MT05 & irregular & undulate & absent & homogeneous & wrinkled & dry & flat & iridescent & small & Blue-green \\
\hline MT07 & circular & entire & absent & homogeneous & rough & dry & flat & iridescent & small & Blue-green \\
\hline \multicolumn{11}{|l|}{ PA01 and PAi } \\
\hline MT10 & circular & entire & absent & homogeneous & smooth & mucoid & flat & opaque & small & yellow \\
\hline
\end{tabular}

${ }^{\mathrm{a}} \mathrm{MF10}$ was observed in both PA01 and PAI2 populations.

phenotypically diversified populations, $1 \mathrm{mg} / \mathrm{L}$ of ciprofloxacin was able to eradicate the diversified ATCC population, but did not affect PAI diversified population (Fig. 4C). Interestingly, a significantly load reduction of the non-diversified ASM-grown PAO1 was obtained. The outcomes related to the application of $1 \mathrm{mg} / \mathrm{L}$ of ciprofloxacin at day 5 against diversified populations were not improved when ciprofloxacin concentration was raised to $4 \mathrm{mg} / \mathrm{L}$ (Fig. 4D). Improvements were only noticed in the non-diversified PAO1 population that was eradicated. In

A

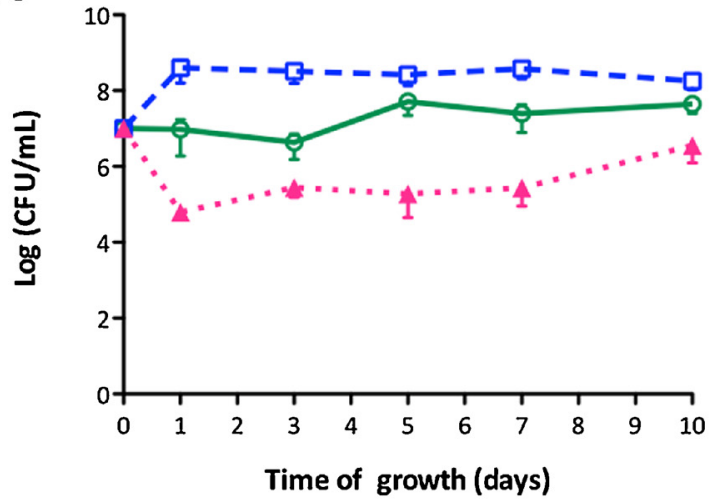

C

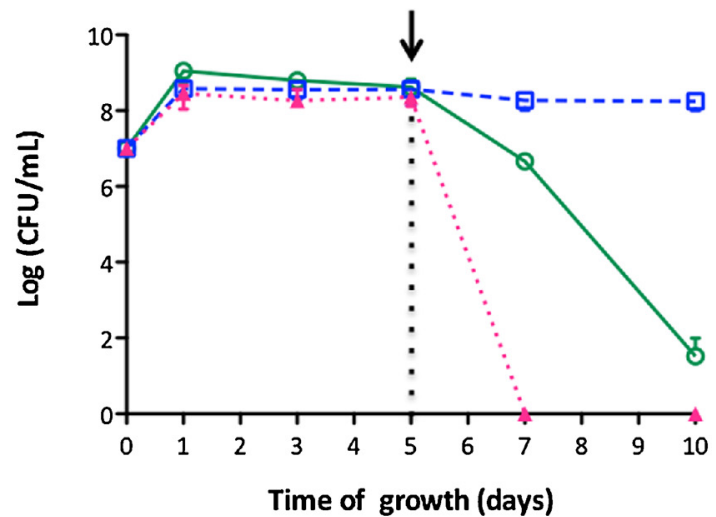

overall, the increase of ciprofloxacin dosage could be beneficial for $P$. aeruginosa eradication from ASM. However, this improvement of ciprofloxacin action did not seem to be related with the phenotypic diversification of ASM-grown populations at this early stage of infection development because both non- and diversified populations, PAO1 and ATCC respectively, were eradicated using 1 and $4 \mathrm{mg} / \mathrm{L}$.

In what concerns ciprofloxacin action, determined by the number of days needed to provoke bacterial eradication, its efficacy was not

B

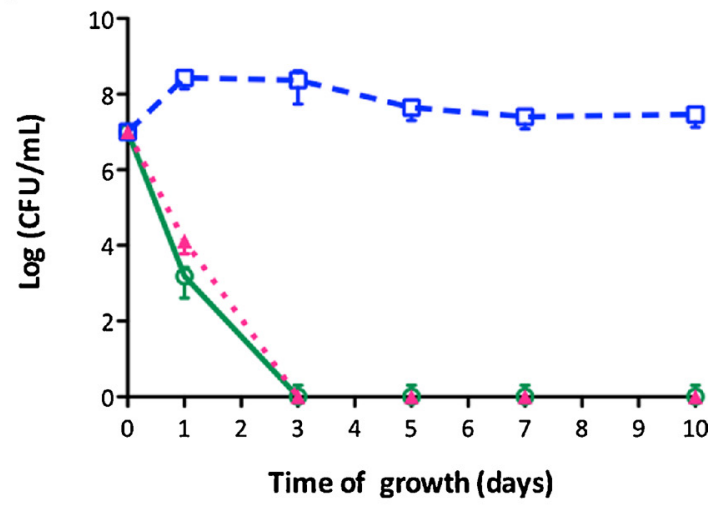

D

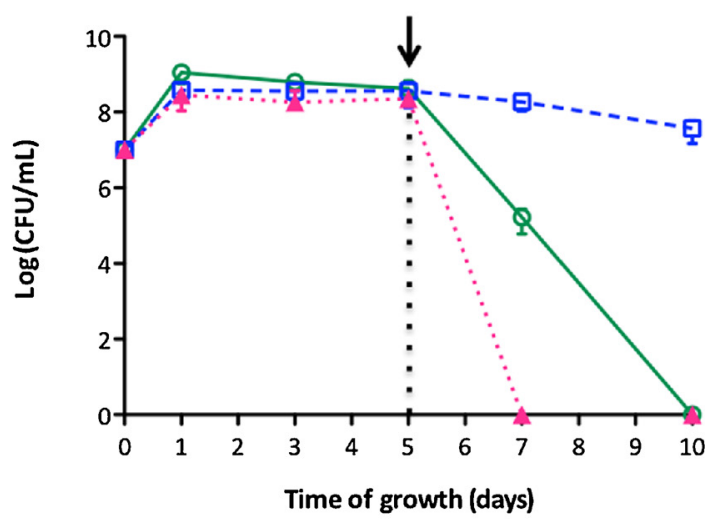

\section{- PA01 -A-ATCC $\approx$ PAI}

Fig. 4. Time killing curves of ciprofloxacin treatments. (A) Ciprofloxacin treatments using $1 \mathrm{mg} / \mathrm{L}$ and (B) using $4 \mathrm{mg} / \mathrm{L}$ over 10 days (applied at day 0 ) against nonphenotypic diversified ASM-grown P. aeruginosa populations. (C) Ciprofloxacin treatments using $1 \mathrm{mg} / \mathrm{L}$ and (D) using $4 \mathrm{mg} / \mathrm{L}$ started at day 5 (indicated by the arrow) over 5 days against ASM-grown phenotypic non- and diversified P. aeruginosa populations. 
impaired by the occurrence of phenotypic diversity of populations. An increase in ciprofloxacin efficacy was noticed when dosages of 1 and $4 \mathrm{mg} / \mathrm{L}$ were applied against ATCC diversified population, as eradication was achieved in two days. However, ciprofloxacin efficacy did not change against non- and diversified PAI populations, where eradication did not occur. These different ciprofloxacin efficacy results allowed to verify that phenotypic diversification of ASM populations did not impair ciprofloxacin efficacy at this early stage.

Altogether, our data evidenced that the efficacy of the first ciprofloxacin treatments against early CF-adapted $P$. aeruginosa could be not impaired by phenotypic diversification, but it could be affected by the $P$. aeruginosa strain susceptibility. Indeed, WTM of PAO1 and ATCC, MT02 and MT01 respectively, were susceptible to ciprofloxacin and their ASM-grown populations were the most sensitive to ciprofloxacin whatever the antibiotic treatment approach followed. On the other hand, PAI was the most resistant strain, as no eradication or even bacterial load reduction was achieved using all ciprofloxacin treatments, and its WTM (MT14) was resistant to ciprofloxacin. Our results also pointed out that ASM colonization by mucoid strains did not seem to represent increased risk of accelerated infections development or diminish antibiotic eradication as previously stated (Li et al., 2005; Schelstraete et al., 2013).

\subsection{P. aeruginosa populations response to ciprofloxacin treatments}

Despite the still positive result of the antibiotic treatments over early ASM-grown $P$. aeruginosa populations, ciprofloxacin seemed to have the potential to trigger clonal diversification into new colony variants. When PAO1 populations were subjected to ciprofloxacin treatment, new colony morphotypes emerged, including MT17 - a moist variant - and MT10 - a mucoid SCV (Fig. 2B and C) that were not detected in the non-treated PAO1 population (Fig. 2A). The same trend was observed for ATCC populations with the emergence of MT03, MT15 (SCV) and MT19 (a pinpoint variant - diameter below $1 \mathrm{~mm}$ ). These PAO1 and ATCC colony variants exhibited morphological features very similar to those observed in clinical isolates from CF-associated chronic infections, including mucoid-alike consistency (MT17) and small (MT10 and MT15) and pinpoint size (MT19) (Table 1). Interestingly, SCV and pinpoint variants from PAO1 and ATCC emerged with relevant estimated prevalence within populations (Figure S1) that suffered a bacterial load reduction caused by ciprofloxacin action.

The increase of ciprofloxacin dosage applied against non- and diversified ASM-grown populations did not seem increase the number of morphotypes identified within populations. Population diversity only increased from 4 to 5 morphotypes when the amount of ciprofloxacin applied against PAI diversified populations at day 5 increased from 1 to $4 \mathrm{mg} / \mathrm{L}$.

The expected absence of mutators in ASM-grown P. aeruginosa PAO1 and ATCC populations exposed to ciprofloxacin treatments (data not shown) led to propose that sub-inhibitory concentrations of ciprofloxacin could be the driving forces underlying clonal diversification into possible "fitter" variants. On the other hand, ciprofloxacin treatments did not prompt clonal diversification in ASM-grown PAI populations as no new colony morphotypes were observed (Fig. 2). WMT and all SCV detected along ciprofloxacin treatments were identical to those observed in the non-treated ASM-grown PAI population. The tolerance of WMT and all SCV to ciprofloxacin (Table 2) may substantiate that there is no need of this bacterial population in diversify into new variants to counteract antibiotic stress.

\subsection{Pathogenic potential of the colony morphology-associated bacteria}

Data derived from our study highlighted that early phenotypic diversity of ASM-grown $P$. aeruginosa populations did not seem to interfere with ciprofloxacin efficacy (Fig. 4), not corroborating previous findings that a diverse population has more chances to persist under
Table 2

Antibiotic susceptibility of the colony morphotypes-associated bacteria detected in ASM-grown $P$. aeruginosa populations.

\begin{tabular}{lllllllllll}
\hline & \multicolumn{1}{c}{ Antibiotic susceptibility } & & & & & \\
\cline { 2 - 9 } & CIP & CN & TOB & AK & IMI & ATM & CAZ & FEP & TTC & PRL \\
\hline PA01 & & & & & & & & & & \\
MT02 (WMT) & S & S & S & S & S & S & S & S & S & S \\
MT17 & S & S & S & S & S & S & S & S & I & S \\
MT10 & I & S & S & S & S & S & S & S & S & S \\
ATCC & & & & & & & & & & \\
MT01 (WMT) & S & S & S & S & S & S & S & S & S & S \\
MT12 & S & S & S & S & S & S & S & S & S & S \\
MT03 & S & S & S & S & S & S & S & S & S & S \\
MT19 & S & S & S & S & I & S & S & S & S & S \\
MT15 & R & S & S & S & I & S & S & S & S & S \\
PAI & & & & & & & & & & \\
MT14 (WMT) & R & S & S & S & S & I & S & S & I & S \\
MT04 & R & S & S & S & S & S & S & S & S & S \\
MT05 & R & S & S & S & S & I & S & S & I & S \\
MT07 & R & S & S & S & S & I & S & S & I & S \\
MT10 & I & S & S & S & S & S & S & S & S & S \\
\hline
\end{tabular}

$\mathrm{S}$ - susceptible; I - intermediate resistant; $\mathrm{R}$ - resistant.

CIP - ciprofloxacin; CN - gentamicin; TOB - tobramycin; AK- amikacin; IMI imipenem; ATM - aztreonam; CAZ - ceftazidime; FEP - cefepime; TTC - ticarcillin/clavulanic acid ; PRL - piperacillin.

stressful conditions, as antibiotic treatments (Boles et al., 2004; Boles and Singh, 2008; Ashish et al., 2013). To better understand these outcomes, the pathogenic potential of all colony variants-associated bacteria isolated from the ASM-grown $P$. aeruginosa populations, different from WMT, was investigated including the antibiotic susceptibility and the most relevant virulence factors expression in CF context. Our results revealed that the colony morphotype-associated bacteria expressed different combinatorial virulence determinants and antibiotic susceptibility profiles, suggesting some biological fitness of $P$. aeruginosa populations that harbour distinct colony variants. The colony variantsassociated bacteria of $P$. aeruginosa exhibited variable ability to form biofilms (Fig. 5A) and pigment production (pyocyanin and pyomelanin) and no alteration in hemolysin, pyoverdine and pyorubrin production, auxotrophy (Table 3) and swarming and twitching motility (Fig. 5C and D). We must highlight the particular increased ability of PAI morphotypes to form biofilms with impressive amount of biomass (Fig. 5A), which may explain the inefficacy of ciprofloxacin over PAI populations.

Among the variability of virulence factors expression, the emergent colony morphotype-associated bacteria exhibited impaired swimming motility in contrast to WMT (Fig. 5B). These results seem to indicate that $P$. aeruginosa evolved to a non-swimming phenotype and this observable characteristic may be an indicator of $P$. aeruginosa adaptation to $\mathrm{CF}$ environment and of the onset of infection development. However, to unambiguously establish this feature as a hallmark of CF early infections, more $P$. aeruginosa strains need to be studied, as well as using in vitro models mimicking as much as possible the heterogeneous characteristics of the inflamed and polymicrobially colonized CF lungs.

Regarding antibiotic susceptibility, the majority of the new colony variants-associated bacteria exhibited antibiotic resistance to ciprofloxacin either by heritance, such as PAI morphotypes, or by exposure to ciprofloxacin, such as MT10 and MT15 (Table 2). The exposure to ciprofloxacin might also contribute to the emergence of colony variants-associated bacteria with augmented resistance against no-related antibiotics including ticarcillin, as evidenced by MT17, and imipenem, exhibited by MT15 and MT19.

In overall, the emergent colony variants-associated bacteria still preserve several WMT features, but our results pointed out that important pathogenic traits for infection development were altered, such as resistance to antibiotics and impaired swimming motility. 
A

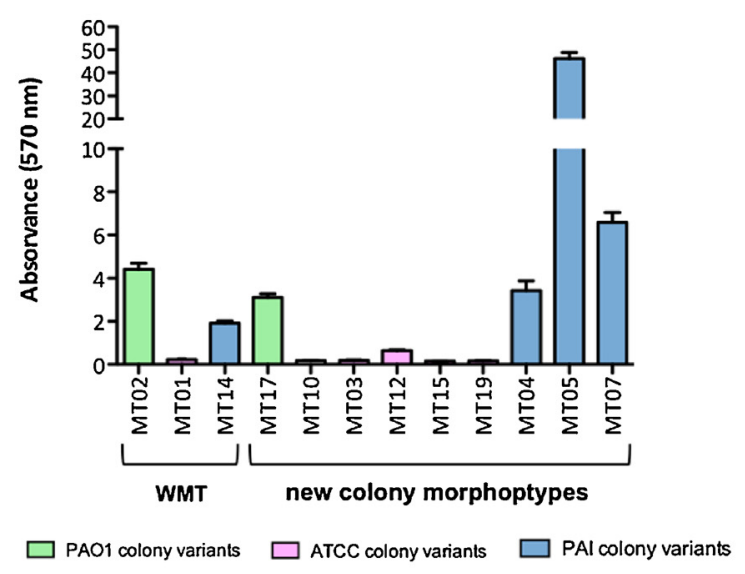

C

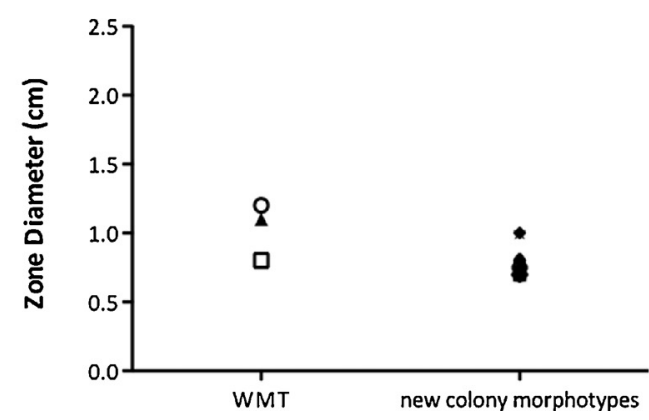

B

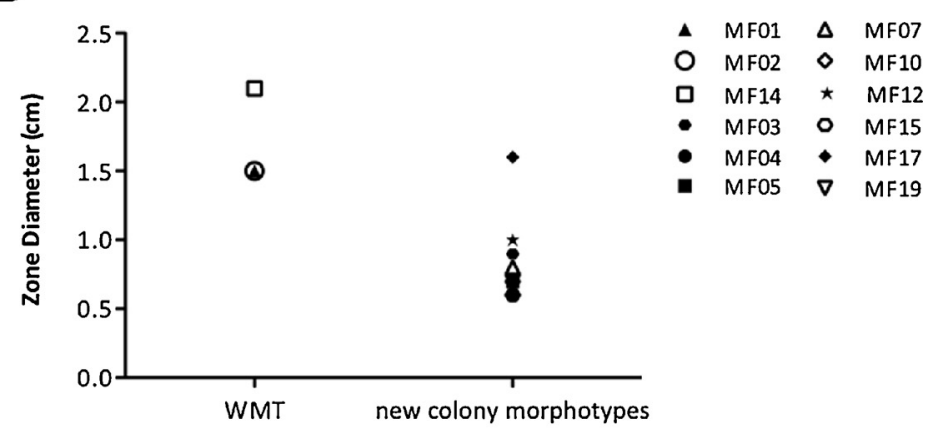

D

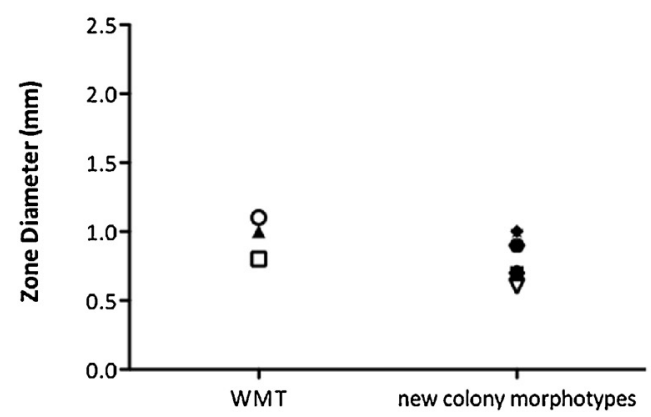

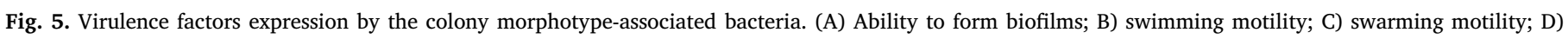

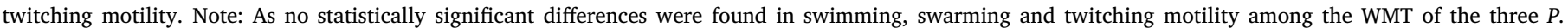
aeruginosa strains and among the several new colony morphotypes, the error bars were omitted to facilitate the results reading.

\section{Discussion}

Understanding $P$. aeruginosa evolution and diversification along the course of infections in CF lungs is of upmost importance, mainly for the decision of the antibiotic treatments to be administered to patients. Nevertheless, those evolutionary processes at the early stage of infection are poorly characterized, remaining unknown what happens immediately after $P$. aeruginosa lungs colonization. The less amount of information concerning early infection stages is mainly due to the need of continuous sampling of the patient sputum until detection of $P$. aeruginosa colonization. The difficulty of determining with precision this onset of colonization limits the ability to define when $P$. aeruginosa starts its adaptation and, thus, the scrutiny of which are the new emergent CF-adapted phenotypes and what are the driving forces of that diversification. Therefore, in vitro studies have a crucial role to provide valuable insights into what occurs to $P$. aeruginosa after enter in CF lungs.

Clonal diversification has been reported as a source of phenotypic

Table 3

Virulence factors expression exhibited by emergent colony morphotypes-associated bacteria detected in ASM-grown P. aeruginosa populations in comparison to WMT.

\begin{tabular}{|c|c|c|c|c|c|c|}
\hline & \multicolumn{6}{|c|}{ Virulence factors expression } \\
\hline \multicolumn{7}{|l|}{ PA01 } \\
\hline MT17 & $+*$ & - & - & - & - & - \\
\hline MT10 & - & - & - & - & - & - \\
\hline \multicolumn{7}{|l|}{ ATCC } \\
\hline MT01 (WMT) & - & - & - & - & + & - \\
\hline MT19 & - & - & - & - & - & - \\
\hline MT15 & - & - & - & - & - & - \\
\hline \multicolumn{7}{|l|}{ PAI } \\
\hline MT14 (WMT) & - & - & - & ++ & - & - \\
\hline MT04 & - & - & - & + & + & - \\
\hline MT05 & - & - & - & + & + & - \\
\hline MT07 & - & - & - & + & + & - \\
\hline
\end{tabular}

*slight hemolysin activity. 
heterogeneity with a role in bacterial adaptation to new environments (Rakhimova et al., 2009; Klockgether et al., 2013; Browall et al., 2014; Markussen et al., 2014). In CF disease, it is well-known that clonal diversification has a fundamental role in chronic infection development and persistence (Klockgether et al., 2013; Markussen et al., 2014; Sousa and Pereira, 2014). In contrast, it is unclear whether clonal diversification can also occur after $\mathrm{CF}$ lungs colonization and if it helps the development of early infections. Moreover, it is unknown whether the occurrence of early phenotypic diversification could be the cause of the failure of the first anti-pseudomonal antibiotic treatments and pave the way for the faster development of chronic infections. To clarify these issues, our study aimed to analyse the early adaptation events of three distinct $P$. aeruginosa populations after simulating in vitro first colonization of CF lungs.

The in vitro model used in our study was constructed based on the ASM model described by Sriramulu et al. (2005). ASM provides quite similar chemical and nutritional conditions to those found in CF lungs, however in the scope of our study the use of ASM was not considered enough when a complex evolutionary pathway of this pathogenic agent is being investigated. $P$. aeruginosa evolutionary pathway in CF lungs towards chronic infections frequently takes several months or even years. Therefore, it is important to let bacteria grow in ASM as long as the in vitro conditions would permit in order to bacteria trigger the mechanisms that this bacterium would use in in vivo scenario. So, in our study, $P$. aeruginosa was allowed to grow in ASM over 10 days to provide time to bacteria initiate their adaptation to this new environment. Using this experimental setup, each evolutionary step of $P$. aeruginosa after ASM colonization and at early stage of infection could be monitored.

Our study provided evidences that ASM-grown $P$. aeruginosa populations evolved along time generating diverse sub-populations straindependent, noticeable by the distinct colony morphotypes. So, we speculated that clonal diversification could start occurring immediately after CF lungs colonization. Nevertheless, WMT of PAO1 strain did not diversify after in vitro simulated $\mathrm{CF}$ lungs colonization and along 10 days. This evidence led us to consider that WMT PAO1 was prepared to grow in ASM with no need to generate "fitter" variants. In this situation, we hypothesized that diversification might occur triggered by alterations in $\mathrm{CF}$ environment, such as antibiotic treatments.

Each $P$. aeruginosa ASM-grown population was composed by distinct colony morphotypes despite all strains belong to the same bacterial species. This intra-strain diversity may be explained by the different function of the central elements in genome, despite the genes involved in regulation were highly conserved. This instability of the core elements of regulation is sufficient to generate distinct clonal diversification and adaptive pathways among strains (Ryall et al., 2012).

The presence of mutators within populations can accelerate $P$. aeruginosa $\mathrm{CF}$-adaptation through high mutation rates up to 1000 -fold than non-mutator phenotypes (Oliver et al., 2000; Mena et al., 2008; Rodriguez-Rojas et al., 2012). Despite mutators are known to be not efficient to establish a primary infection (Montanari et al., 2007), their presence in the ASM-grown $P$. aeruginosa populations was investigated to unequivocally discard them as a cause of diversity. As expected, mutators were not detected that led to speculate that the driving forces for the observed diversification might be related with niche specialization. Markussen et al. (2014) also found initial infecting strain diversification into distinct sublineages immediately after initial lungs colonization and proposed niche partitioning as the driving force for phenotypic diversification. CF sputum is known to be intrinsically heterogeneous and highly structured environment with multiple compartments where distinct environmental conditions could be found, for instance, different oxygen and nutrient availability, antibiotic molecules and immune cells (Worlitzsch et al., 2002; Hoffman et al., 2010). Bacteria fill in these distinct ecological compartments leading to niche specialization and, consequently, to population diversity. ASM in the 24-well plates had a depth of approx. $1.4 \mathrm{~cm}$ that incubating statically along 10 days might has allowed to mimic different oxygen diffusion and provoking different availability. Moreover, during our experiments several visible aggregates within ASM suspensions with distinct size and apparent density were observed that could be indicative of aggregated or biofilm formation. These possible distinct bacterial lifestyles (free living and biofilm) could result in different access to nutrients. So, these observations led us to hypothesize that ASM could also mimic niche specialization of $\mathrm{CF}$ sputum. The rapid diversification of $P$. aeruginosa after initial ASM colonization in our study could be caused by the existence of several niches within ASM determined by the different oxygen and/or nutrient concentrations in which bacteria grow leading to several sub-populations. The topic of niche specialization within $\mathrm{CF}$ sputum is very difficult to unravel due to the lack of both in vitro and in vivo studies. The results of our study reinforced that more efforts must be addressed to clarify the role of niche specialization since early stages of infection.

According the 'Insurance Hypothesis', (more) diversity within a population enhances its functioning against a wide range of adverse environmental fluctuations increasing thus the chances of survival [43]. Therefore, we hypothesized that early phenotypic diversification could be the cause of the failure of the first anti-pseudomonal treatments in $P$. aeruginosa eradication from CF lungs. Our results demonstrated that early phenotypic diversification of $P$. aeruginosa did not represent a critical issue for antibiotic treatments, at least, at early infection stage. The efficacy of ciprofloxacin to eradicate ASM-grown populations seemed to be more affected by $P$. aeruginos a antibiotic susceptibility than by population diversity. ASM-grown PAI populations resisted to all ciprofloxacin treatments probably due to WMT resistance to ciprofloxacin, in contrast to PAO1 and ATCC ASM-populations that were susceptible to ciprofloxacin similarly to their WMT. PAO1 strain has no gene islands encoding relevant virulence factors to tolerate aggressive concentrations of antibiotic which might explained its facilitated eradication (Stover et al., 2000). Early colonization of the CF airways with mucoid strains is often associated with an accelerated rate of decline in pulmonary function (Li et al., 2005; Schelstraete et al., 2013). According our results, the mucoidy feature of the ATCC strain did not appear to confer any additional biological advantage to $P$. aeruginosa to adapt to $\mathrm{CF}$ environment or protection against the antimicrobial activity of ciprofloxacin. Mayer-Hamblett et al. (2014a,b) did not find as well an association of mucoidy with severe infection development. In contrast, first $P$. aeruginosa acquisition from non-CF individual seemed to represent a risk factor because PAI populations were not eradicated. These assumptions should be tested with other non- and mucoid strains.

Although phenotypic diversity did not have impact on ciprofloxacin efficacy, antibiotic concentration revealed to be important in early eradication of $P$. aeruginosa. Beyond sub-biofilm inhibitory concentrations of ciprofloxacin did not demonstrate to be effective in $P$. aeruginosa eradication, they seemed to promote clonal diversification into new variants. The new emergent colony variants exhibited some typical traits of chronic isolates (Sousa and Pereira, 2014), such as small size, the SCV, (MT10, MT15 and MT19) and mucoid consistency (MT10) or mucoid-trend consistency (MT17). Moreover, these variants exhibited some altered antibiotic susceptibility to ciprofloxacin and to non-related antibiotics. Other studies also found that sub-inhibitory concentrations of antibiotic can drive diversification and selection of populations with distinct antibiotic resistance levels in response to different ciprofloxacin concentrations (Macia et al., 2006; Wright et al., 2013).

The expected absence of mutators within ASM-grown populations under sub-inhibitory ciprofloxacin treatments supported the assumption that sub-inhibitory dosages might function as driving forces for clonal diversification into "fitter" variants. Indeed, sub-inhibitory or sub-lethal concentrations of antibiotics can act as signalling molecules regulating gene transcription, physiology and virulence potential (Shen et al., 2008; Skindersoe et al., 2008; Cummins et al., 2009; Douglas et al., 2009) inducing mutations and recombinations and, consequently, 
supporting the emergence of phenotypic variants (Kohanski et al., 2010; Rodriguez-Rojas et al., 2012; Wright et al., 2013). The emergence of colony morphotypes with mucoid consistency (MT10), small (MT10 and MT15) and pinpoint size (MT19) under antibiotic pressure at early stage could be understood as an attempt of $P$. aeruginosa to adapt to the harmful conditions imposed. We hypothesized that these new-arise colony variants started to exhibit some phenotypic characteristics distinct from those displayed by WMT to escape the toxic action of ciprofloxacin, augmenting thus their bacterial pathogenicity.

The long-term in vitro maintenance of $P$. aeruginosa in ASM provided a valuable opportunity to detect one of its first steps of the evolutionary pathway in CF lungs. Although colony morphotypes-associated bacteria still preserve several WMT characteristics, the first steps of microevolution and adaptation were noticed through the increased ability to form biofilms that some colony morphotypes demonstrated, but, more importantly, the evident impaired swimming motility that the majority of them exhibited. Swimming motility is promoted by flagella and their lack inhibit swimming movement facilitating other further phenotypic adaptations, such as aggregation (biofilm formation) that confer protection against to antibiotics and host immune molecules (Mahenthiralingam et al., 1994; Staudinger et al., 2014). MayerHamblett et al. (2014a,b) found an association between defective swimming ability and pulmonary exacerbations, worsening symptoms and declining lung function, the common signs of infection development (Bilton et al., 2011; Mowat et al., 2011; Stressmann et al., 2011). Therefore, we hypothesized the emergence of colony variants-associated bacteria with impaired swimming motility detected in our study may be at the root of the first $P$. aeruginosa infections and the beginning of lung function decline in CF patients. Several other studies have described defective swimming ability as a characteristic of CF chronic isolates (Mahenthiralingam et al., 1994; Manos et al., 2013; Staudinger et al., 2014) which reinforce our assumption that impaired swimming motility could be considered one of the early adaptationsof $P$. aeruginosa towards infection development. Defective swimming motility could be a potential disease marker with great impact on clinical diagnosis. As swimming measures can be performed in a reproducible and quantitative way, an easily diagnosis of early $P$. aeruginosa adaptation could be achieved. Therefore, it is urgent that more studies address the early adaptive events that $P$. aeruginosa undergoes in CF lungs to strengthen this suggestion of early adaptation marker.

Our long-term in vitro maintenance model represent a step forward towards in vivo scenario and it provided valuable insights about the early evolutionary steps of $P$. aeruginosa in CF environment. However, it did not fully mimic the complex environment of CF lungs. Therefore, more studies using improved in vitro models, mimicking as much as possible the heterogeneous characteristics of CF lungs, and encompassing more $P$. aeruginosa strains, mono- or co-cultured with other bacteria, need to be carried out to unequivocally establish early clonal diversification occurrence and impaired swimming motility as the stamp of the early onset $P$ aeruginosa infection in CF patients.

\section{Conclusions}

Our results evidenced that clonal diversification typically detected in chronic infection stages, could begin immediately after CF lungs colonization by $P$. aeruginosa in a strain-dependent way. We hypothesized that this diversification could be driven by niche specialization in which bacteria organize themselves spatially into distinct ecological niches of CF sputum. However, at this early stage of infection, clonal diversification did not have significant impact on ciprofloxacin effectiveness since biofilm inhibitory dosages would be applied. The use of biofilm sub-inhibitory concentrations, besides not be effective in $P$. aeruginosa eradication, can trigger the emergence of colony variants exhibiting characteristics very alike to chronic isolates, including SCV, pinpoint and mucoid variants resistant to antibiotics. Our evidences are of vital clinical significance because they highlighted that inadequate first antibiotic dosages, beyond do not treat infection, may select resistant bacteria, contributing to infection resilience to subsequent antimicrobial interventions. The analysis of the pathogenic potential of all emergent colony variants-associated bacteria, isolated from the several ASM experiments, revealed that, comparing to WMT, $P$. aeruginosa evolve to a non-swimming phenotype. These findings led us to suggest that impaired swimming motility could be the hallmark of the onset of early infection and one of the very first steps of the long and complex evolutionary pathway towards chronic infections. Thus, limited swimming motility could be a potential candidate for CF early infection biomarker, hypothesis that needs to be tested by analysing the phenotypic and pathogenic behaviour of more $P$. aeruginosa isolates.

The outcomes of our study will certainly assist the accurate design of the first anti-pseudomonal antimicrobial treatments and increase their successful eradication rates. Despite the usefulness of the in vitro long-term growth of $P$. aeruginosa used in this study, this in vitro model has only allowed the detection of some of the first evolutionary steps that are at the root of the long and complex pathway towards chronic infection. CF lungs are extremely complex environment and other characteristics such as excessive inflammation response and their polymicrobial nature should be simulated in the in vitro studies to obtain results closer to the in vivo onset of infection. Only by this way we will able to understand the early $P$. aeruginosa adaptation to CF lungs and the events underlying the transition of early infection to chronic infection.

\section{Acknowledgements}

This work was supported by the Portuguese Foundation for Science and Technology (FCT) under the scope of the strategic funding of UID/ BIO/04469 unit and COMPETE 2020 (POCI-01-0145-FEDER-006684) and BioTecNorte operation (NORTE-01-0145-FEDER-000004) funded by the European Regional Development Fund under the scope of Norte2020 - Programa Operacional Regional do Norte. The authors also acknowledge COMPETE2020 and FCT for the project POCI-01-0145FEDER-029841.

\section{Appendix A. Supplementary data}

Supplementary material related to this article can be found, in the online version, at doi:https://doi.org/10.1016/j.ijmm.2018.10.003.

\section{References}

Ashish, A., Paterson, S., Mowat, E., Fothergill, J.L., Walshaw, M.J., Winstanley, C., 2013. Extensive diversification is a common feature of Pseudomonas aeruginosa populations during respiratory infections in cystic fibrosis. J. Cyst. Fibros. 12, 790-793.

Bilton, D., Canny, G., Conway, S., Dumcius, S., Hjelte, L., Proesmans, M., Tummler, B., Vavrova, V., De Boeck, K., 2011. Pulmonary exacerbation: towards a definition for use in clinical trials. Report from the EuroCareCF Working Group on outcome parameters in clinical trials. J. Cyst. Fibros. 10 (Suppl. 2), S79-81.

Bittar, F., Richet, H., Dubus, J.C., Reynaud-Gaubert, M., Stremler, N., Sarles, J., Raoult, D., Rolain, J.M., 2008. Molecular detection of multiple emerging pathogens in sputa from cystic fibrosis patients. PLoS One 3, e2908.

Boles, B.R., Singh, P.K., 2008. Endogenous oxidative stress produces diversity and adaptability in biofilm communities. Proc. Natl. Acad. Sci. U. S. A. 105, 12503-12508.

Boles, B.R., Thoendel, M., Singh, P.K., 2004. Self-generated diversity produces' insurance effects' in biofilm communities. Proc. Natl. Acad. Sci. U. S. A. 101, 16630-16635.

Bragonzi, A., Paroni, M., Nonis, A., Cramer, N., Montanari, S., Rejman, J., Di Serio, C., Doring, G., Tummler, B., 2009. Pseudomonas aeruginosa microevolution during cystic fibrosis lung infection establishes clones with adapted virulence. Am. J. Respir. Crit. Care Med. 180, 138-145.

Browall, S., Norman, M., Tangrot, J., Galanis, I., Sjostrom, K., Dagerhamn, J., Hellberg, C., Pathak, A., Spadafina, T., Sandgren, A., Battig, P., Franzen, O., Andersson, B., Ortqvist, A., Normark, S., Henriques-Normark, B., 2014. Intraclonal variations among Streptococcus pneumoniae isolates influence the likelihood of invasive disease in children. J. Infect. Dis. 209, 377-388.

Burns, J.L., Gibson, R.L., McNamara, S., Yim, D., Emerson, J., Rosenfeld, M., Hiatt, P., McCoy, K., Castile, R., Smith, A.L., Ramsey, B.W., 2001. Longitudinal assessment of Pseudomonas aeruginosa in young children with cystic fibrosis. J. Infect. Dis. 183, 444-452. 
Ciofu, O., Tolker-Nielsen, T., Jensen, P.O., Wang, H., Hoiby, N., 2014. Antimicrobial resistance, respiratory tract infections and role of biofilms in lung infections in cystic fibrosis patients. Adv. Drug Deliv. Rev.

Clark, S.T., Diaz Caballero, J., Cheang, M., Coburn, B., Wang, P.W., Donaldson, S.L., Zhang, Y., Liu, M., Keshavjee, S., Yau, Y.C., Waters, V.J., Elizabeth Tullis, D., Guttman, D.S., Hwang, D.M., 2015. Phenotypic diversity within a Pseudomonas aeruginosa population infecting an adult with cystic fibrosis. Sci. Rep. 5, 10932.

Clinical and Laboratory Standards Institute, 2007. Performance Standards for Antimicrobial Susceptibility Testing; Seventeenth Informational Supplement M100S17. Clinical and Laboratory Standards Institute, Wayne, PA, USA.

Clinical and Laboratory Standards Institute, 2012. Performance Standards for Antimicrobial Disk Susceptibility Tests - Twenty-second Informational Supplement M100-S22. Clinical and Laboratory Standards Institute, Wayne, PA, USA.

Clinical and Laboratory Standards Institute, 2018. Performance Standards for Antimicrobial Susceptibility Testing - 28th. CLSI Supplement M100. Clinical and Laboratory Standards Institute, Wayne, PA, USA.

Coburn, B., Wang, P.W., Diaz Caballero, J., Clark, S.T., Brahma, V., Donaldson, S., Zhang, Y., Surendra, A., Gong, Y., Elizabeth Tullis, D., Yau, Y.C., Waters, V.J., Hwang, D.M., Guttman, D.S., 2015. Lung microbiota across age and disease stage in cystic fibrosis. Sci. Rep. 5, 10241.

Coutinho, H.D., Falcao-Silva, V.S., Goncalves, G.F., 2008. Pulmonary bacterial pathogens in cystic fibrosis patients and antibiotic therapy: a tool for the health workers. Int. Arch. Med. 1, 24.

Cummins, J., Reen, F.J., Baysse, C., Mooij, M.J., O'Gara, F., 2009. Subinhibitory concentrations of the cationic antimicrobial peptide colistin induce the pseudomonas quinolone signal in Pseudomonas aeruginosa. Microbiology 155, 2826-2837.

Deziel, E., Comeau, Y., Villemur, R., 2001. Initiation of biofilm formation by Pseudomonas aeruginosa 57RP correlates with emergence of hyperpiliated and highly adherent phenotypic variants deficient in swimming, swarming, and twitching motilities 1. J. Bacteriol. 183, 1195-1204.

Douglas, T.A., Brennan, S., Gard, S., Berry, L., Gangell, C., Stick, S.M., Clements, B.S., Sly, P.D., 2009. Acquisition and eradication of $P$. aeruginosa in young children with cystic fibrosis. Eur. Respir. J. 33, 305-311.

Emiralioglu, N., Yalcin, E., Meral, A., Sener, B., Dogru, D., Ozcelik, U., Kiper, N., 2016. The success of the different eradication therapy regimens for Pseudomonas aeruginosa in cystic fibrosis. J. Clin. Pharm. Ther. 41, 419-423.

Flume, P.A., Mogayzel Jr, P.J., Robinson, K.A., Goss, C.H., Rosenblatt, R.L., Kuhn, R.J., Marshall, B.C., 2009. Cystic fibrosis pulmonary guidelines: treatment of pulmonary exacerbations. Am. J. Respir. Crit. Care Med. 180, 802-808.

Folkesson, A., Jelsbak, L., Yang, L., Johansen, H.K., Ciofu, O., Hoiby, N., Molin, S., 2012. Adaptation of Pseudomonas aeruginosa to the cystic fibrosis airway: an evolutionary perspective. Nat. Rev. Microbiol. 10, 841-851.

Hassett, D.J., Sutton, M.D., Schurr, M.J., Herr, A.B., Caldwell, C.C., Matu, J.O., 2009. Pseudomonas aeruginosa hypoxic or anaerobic biofilm infections within cystic fibrosis airways. Trends Microbiol. 17, 130-138.

Haussler, S., 2004. Biofilm formation by the small colony variant phenotype of Pseudomonas aeruginosa. Environ. Microbiol. 6, 546-551.

Haussler, S., Tummler, B., Weissbrodt, H., Rohde, M., Steinmetz, I., 1999. Small-colony variants of Pseudomonas aeruginosa in cystic fibrosis. Clin. Infect. Dis. 29, 621-625.

Haussler, S., Ziegler, I., Lottel, A., von Gotz, F., Rohde, M., Wehmhohner, D., Saravanamuthu, S., Tummler, B., Steinmetz, I., 2003. Highly adherent small-colony variants of Pseudomonas aeruginosa in cystic fibrosis lung infection. J. Med. Microbiol. 52, 295-301.

Høiby, N., Ciofu, O., Bjarnsholt, T., 2010. Pseudomonas aeruginosa biofilms in cystic fibrosis. Future Microbiol. 5, 1663-1674.

Hoffman, L.R., Richardson, A.R., Houston, L.S., Kulasekara, H.D., Martens-Habbena, W., Klausen, M., Burns, J.L., Stahl, D.A., Hassett, D.J., Fang, F.C., Miller, S.I., 2010. Nutrient availability as a mechanism for selection of antibiotic tolerant Pseudomonas aeruginosa within the CF airway. PLoS Pathog. 6, e1000712.

Hogardt, M., Heesemann, J., 2010. Adaptation of Pseudomonas aeruginosa during persistence in the cystic fibrosis lung. Int. J. Med. Microbiol. 300, 557-562.

Jiricny, N., Molin, S., Foster, K., Diggle, S.P., Scanlan, P.D., Ghoul, M., Johansen, H.K., Santorelli, L.A., Popat, R., West, S.A., Griffin, A.S., 2014. Loss of social behaviours in populations of Pseudomonas aeruginosa infecting lungs of patients with cystic fibrosis. PLoS One 9, e83124.

Kahl, B.C., 2014. Small colony variants (SCVs) of Staphylococcus aureus - a bacterial survival strategy. Infect. Genet. Evol. 21, 515-522.

Kirisits, M.J., Prost, L., Starkey, M., Parsek, M.R., 2005. Characterization of colony morphology variants isolated from Pseudomonas aeruginosa biofilms. Appl. Environ. Microbiol. 71, 4809-4821.

Klockgether, J., Miethke, N., Kubesch, P., Bohn, Y.S., Brockhausen, I., Cramer, N., Eberl, L., Greipel, J., Herrmann, C., Herrmann, S., Horatzek, S., Lingner, M., Luciano, L., Salunkhe, P., Schomburg, D., Wehsling, M., Wiehlmann, L., Davenport, C.F., Tummler, B., 2013. Intraclonal diversity of the Pseudomonas aeruginosa cystic fibrosis airway isolates TBCF10839 and TBCF121838: distinct signatures of transcriptome, proteome, metabolome, adherence and pathogenicity despite an almost identical genome sequence. Environ. Microbiol. 15, 191-210.

Kohanski, M.A., DePristo, M.A., Collins, J.J., 2010. Sublethal antibiotic treatment leads to multidrug resistance via radical-induced mutagenesis. Mol. Cell 37, 311-320.

Kreda, S.M., Davis, C.W., Rose, M.C., 2012. CFTR, mucins, and mucus obstruction in cystic fibrosis. Cold Spring Harb. Perspect. Med. 2, a009589.

Langan, K.M., Kotsimbos, T., Peleg, A.Y., 2015. Managing Pseudomonas aeruginosa respiratory infections in cystic fibrosis. Curr. Opin. Infect. Dis. 28, 547-556.

Li, Z., Kosorok, M.R., Farrell, P.M., Laxova, A., West, S.E., Green, C.G., Collins, J., Rock, M.J., Splaingard, M.L., 2005. Longitudinal development of mucoid Pseudomonas aeruginosa infection and lung disease progression in children with cystic fibrosis.
JAMA 293, 581-588.

Macia, M.D., Borrell, N., Segura, M., Gomez, C., Perez, J.L., Oliver, A., 2006. Efficacy and potential for resistance selection of antipseudomonal treatments in a mouse model of lung infection by hypermutable Pseudomonas aeruginosa. Antimicrob. Agents Chemother. 50, 975-983.

Magalhães, A.P., Lopes, S.P., Pereira, M.O., 2016. Insights into cystic fibrosis polymicrobial consortia: the role of species interactions in biofilm development, phenotype, and response to in-use antibiotics. Front. Microbiol. 7, 2146.

Mahenthiralingam, E., Campbell, M.E., Speert, D.P., 1994. Nonmotility and phagocytic resistance of Pseudomonas aeruginosa isolates from chronically colonized patients with cystic fibrosis. Infect. Immun. 62, 596-605.

Manos, J., Hu, H., Rose, B.R., Wainwright, C.E., Zablotska, I.B., Cheney, J., Turnbull, L., Whitchurch, C.B., Grimwood, K., Harmer, C., Anuj, S.N., Harbour, C., 2013. Virulence factor expression patterns in Pseudomonas aeruginosa strains from infants with cystic fibrosis. Eur. J. Clin. Microbiol. Infect. Dis. 32, 1583-1592.

Markussen, T., Marvig, R.L., Gomez-Lozano, M., Aanaes, K., Burleigh, A.E., Hoiby, N., Johansen, H.K., Molin, S., Jelsbak, L., 2014. Environmental heterogeneity drives within-host diversification and evolution of Pseudomonas aeruginosa. MBio 5, e01592-01514.

Mayer-Hamblett, N., Kronmal, R.A., Gibson, R.L., Rosenfeld, M., Retsch-Bogart, G., Treggiari, M.M., Burns, J.L., Khan, U., Ramsey, B.W., 2012. Initial Pseudomonas aeruginosa treatment failure is associated with exacerbations in cystic fibrosis. Pediatr. Pulmonol. 47, 125-134.

Mayer-Hamblett, N., Rosenfeld, M., Gibson, R.L., et al., 2014a. Pseudomonas aeruginosa in vitro phenotypes distinguish cystic fibrosis infection stages and outcomes. Am. J. Respir. Crit. Care Med. 190, 289-297.

Mayer-Hamblett, N., Ramsey, B.W., Kulasekara, H.D., Retsch-Bogart, G.Z., Morgan, W., Wolter, D.J., Pope, C.E., Houston, L.S., Kulasekara, B.R., Khan, U., Burns, J.L., Miller, S.I., Hoffman, L.R., 2014b. Pseudomonas aeruginosa phenotypes associated with eradication failure in children with cystic fibrosis. Clin. Infect. Dis. 59, 624-631.

Mena, A., Smith, E.E., Burns, J.L., Speert, D.P., Moskowitz, S.M., Perez, J.L., Oliver, A., 2008. Genetic adaptation of Pseudomonas aeruginosa to the airways of cystic fibrosis patients is catalyzed by hypermutation. J. Bacteriol. 190, 7910-7917.

Montanari, S., Oliver, A., Salerno, P., Mena, A., Bertoni, G., Tummler, B., Cariani, L., Conese, M., Doring, G., Bragonzi, A., 2007. Biological cost of hypermutation in Pseudomonas aeruginosa strains from patients with cystic fibrosis. MicrobiologySgm 153, 1445-1454.

Mowat, E., Paterson, S., Fothergill, J.L., Wright, E.A., Ledson, M.J., Walshaw, M.J., Brockhurst, M.A., Winstanley, C., 2011. Pseudomonas aeruginosa population diversity and turnover in cystic fibrosis chronic infections. Am. J. Respir. Crit. Care Med. 183, 1674-1679.

Oliver, A., Canton, R., Campo, P., Baquero, F., Blazquez, J., 2000. High frequency of hypermutable Pseudomonas aeruginosa in cystic fibrosis lung infection. Science 288, 1251-1253.

Peeters, E., Nelis, H.J., Coenye, T., 2007. Comparison of Multiple Methods for Quantification of Microbial Biofilms Grown in Microtiter Plates. Journal of Microbiological Methods In Press, Corrected Proof.

Pires, D., Sillankorva, S., Faustino, A., Azeredo, J., 2011. Use of newly isolated phages for control of Pseudomonas aeruginosa PAO1 and ATCC 10145 biofilms. Res. Microbiol. 162, 798-806.

Qiu, D., Eisinger, V.M., Rowen, D.W., Yu, H.D., 2007. Regulated proteolysis controls mucoid conversion in Pseudomonas aeruginosa. Proc Natl Acad Sci U S A 104, 8107-8112.

Rakhimova, E., Munder, A., Wiehlmann, L., Bredenbruch, F., Tummler, B., 2008. Fitness of isogenic colony morphology variants of Pseudomonas aeruginosa in murine airway infection. PLoS One 3, e1685.

Rakhimova, E., Wiehlmann, L., Brauer, A.L., Sethi, S., Murphy, T.F., Tummler, B., 2009. Pseudomonas aeruginosa population biology in chronic obstructive pulmonary disease. J. Infect. Dis. 200, 1928-1935.

Ratjen, F., 2006. Treatment of early Pseudomonas aeruginosa infection in patients with cystic fibrosis. Curr. Opin. Pulm. Med. 12, 428-432.

Ratjen, F., Munck, A., Kho, P., Angyalosi, G., 2010. Treatment of early Pseudomonas aeruginosa infection in patients with cystic fibrosis: the ELITE trial. Thorax 65, 286-291.

Ratjen, F., Moeller, A., McKinney, M.L., Asherova, I., Alon, N., Maykut, R., Angyalosi, G., 2018. Eradication of early $P$. aeruginosa infection in children $<7$ years of age with cystic fibrosis: the early study. J. Cyst. Fibros.

Rau, M.H., Hansen, S.K., Johansen, H.K., Thomsen, L.E., Workman, C.T., Nielsen, K.F. Jelsbak, L., Hoiby, N., Yang, L., Molin, S., 2010. Early adaptive developments of Pseudomonas aeruginosa after the transition from life in the environment to persistent colonization in the airways of human cystic fibrosis hosts. Environ. Microbiol. 12, 1643-1658.

Rodriguez-Rojas, A., Oliver, A., Blazquez, J., 2012. Intrinsic and environmental mutagenesis drive diversification and persistence of Pseudomonas aeruginosa in chronic lung infections. J. Infect. Dis. 205, 121-127.

Ryall, B., Eydallin, G., Ferenci, T., 2012. Culture history and population heterogeneity as determinants of bacterial adaptation: the adaptomics of a single environmental transition. Microbiol. Mol. Biol. Rev. 76, 597-625.

Schelstraete, P., Haerynck, F., Van Daele, S., Deseyne, S., De Baets, F., 2013. Eradication therapy for Pseudomonas aeruginosa colonization episodes in cystic fibrosis patients not chronically colonized by P. aeruginosa. J. Cyst. Fibros. 12, 1-8.

Shen, L., Shi, Y., Zhang, D., Wei, J., Surette, M.G., Duan, K., 2008. Modulation of secreted virulence factor genes by subinhibitory concentrations of antibiotics in Pseudomonas aeruginosa. J. Microbiol. 46, 441-447.

Skindersoe, M.E., Alhede, M., Phipps, R., Yang, L., Jensen, P.O., Rasmussen, T.B., Bjarnsholt, T., Tolker-Nielsen, T., Hoiby, N., Givskov, M., 2008. Effects of antibiotics 
on quorum sensing in Pseudomonas aeruginosa. Antimicrob. Agents Chemother. 52, 3648-3663.

Sousa, A.M., Pereira, M.O., 2014. Pseudomonas aeruginosa diversification during infection development in Cystic Fibrosis Lungs - a review. Pathogens 3, 680-703.

Sousa, A.M., Machado, I., Nicolau, A., Pereira, M.O., 2013. Improvements on colony morphology identification towards bacterial profiling. J. Microbiol. Methods 95, 327-335.

Sousa, A.M., Lourenço, A., Pereira, M.O., 2015. MorphoCol: an ontology-based knowledgebase for the characterisation of clinically significant bacterial colony morphologies. J. Biomed. Inform. 55, 55-63.

Speert, D.P., Campbell, M.E., Henry, D.A., Milner, R., Taha, F., Gravelle, A., Davidson, A.G., Wong, L.T., Mahenthiralingam, E., 2002. Epidemiology of Pseudomonas aeruginosa in cystic fibrosis in British Columbia. Canada. Am J Respir Crit Care Med 166, 988-993.

Sriramulu, D.D., Lunsdorf, H., Lam, J.S., Romling, U., 2005. Microcolony formation: a novel biofilm model of Pseudomonas aeruginosa for the cystic fibrosis lung. J. Med. Microbiol. 54, 667-676.

Staudinger, B.J., Muller, J.F., Halldorsson, S., Boles, B., Angermeyer, A., Nguyen, D., Rosen, H., Baldursson, O., Gottfreethsson, M., Guethmundsson, G.H., Singh, P.K. 2014. Conditions associated with the cystic fibrosis defect promote chronic Pseudomonas aeruginosa infection. Am. J. Respir. Crit. Care Med. 189, 812-824.

Stepanovic, S., Vukovic, D., Dakic, I., Savic, B., Svabic-Vlahovic, M., 2000. A modified microtiter-plate test for quantification of staphylococcal biofilm formation. J. Microbiol. Methods 40, 175-179.
Stover, C.K., Pham, X.Q., Erwin, A.L., Mizoguchi, S.D., Warrener, P., Hickey, M.J., Brinkman, F.S., Hufnagle, W.O., Kowalik, D.J., Lagrou, M., Garber, R.L., Goltry, L., Tolentino, E., Westbrock-Wadman, S., Yuan, Y., Brody, L.L., Coulter, S.N., Folger, K.R., Kas, A., Larbig, K., Lim, R., Smith, K., Spencer, D., Wong, G.K., Wu, Z., Paulsen, I.T., Reizer, J., Saier, M.H., Hancock, R.E., Lory, S., Olson, M.V., 2000. Complete genome sequence of Pseudomonas aeruginosa PAO1, an opportunistic pathogen. Nature 406, 959-964.

Stressmann, F.A., Rogers, G.B., Marsh, P., Lilley, A.K., Daniels, T.W., Carroll, M.P., Hoffman, L.R., Jones, G., Allen, C.E., Patel, N., Forbes, B., Tuck, A., Bruce, K.D., 2011. Does bacterial density in cystic fibrosis sputum increase prior to pulmonary exacerbation? J. Cyst. Fibros. 10, 357-365.

UK Cystic Fibrosis Trust Infection Control Group, 2004. Pseudomonas AerugInosa Infection in People With Cystic Fibrosis. Suggestions for Prevention and Infection Control, second edition. The UK Cystic Fibrosis Trust, UK.

Workentine, M.L., Sibley, C.D., Glezerson, B., Purighalla, S., Norgaard-Gron, J.C., Parkins, M.D., Rabin, H.R., Surette, M.G., 2013. Phenotypic heterogeneity of Pseudomonas aeruginosa populations in a cystic fibrosis patient. PLoS One 8 e60225.

Worlitzsch, D., Tarran, R., Ulrich, M., Schwab, U., Cekici, A., Meyer, K.C., Birrer, P., Bellon, G., Berger, J., Weiss, T., Botzenhart, K., Yankaskas, J.R., Randell, S., Boucher, R.C., Doring, G., 2002. Effects of reduced mucus oxygen concentration in airway Pseudomonas infections of cystic fibrosis patients. J. Clin. Invest. 109, 317-325.

Wright, E.A., Fothergill, J.L., Paterson, S., Brockhurst, M.A., Winstanley, C., 2013. Subinhibitory concentrations of some antibiotics can drive diversification of Pseudomonas aeruginosa populations in artificial sputum medium. BMC Microbiol. 13, 170. 